\title{
Stem cell treatment of dystrophic dogs
}

\author{
Arising from: M. Sampaolesi et al. Nature 444, 574-579 (2006)
}

Human muscular dystrophies are devastating and incurable inherited diseases. Hopes of progress towards therapy of muscular dystrophies were aroused when Sampaolesi et al. ${ }^{1}$ reported "extensive recovery of dystrophin expression, normal muscle...function", and "remarkable clinical amelioration" in golden retriever muscular dystrophy dogs treated with 'mesoangioblasts'. Here I re-examine their results, showing how their assessments might be flawed and their conclusions overstated. Further studies will be required to evaluate fully the clinical potential of this work.

My first concern is that control and test dogs were not matched for disease characteristics at the start of treatment to avoid inadvertently biased groupings in small experimental cohorts, where extensive individual variations exist. Also, the authors' evaluations were not blinded. For example, their Supplementary Videos ${ }^{1}$ seem to show greater human encouragement of treated compared with untreated dogs, so functional recovery may not be independently verifiable by viewers, especially the "striking improvement of motility" in the older dogs treated with mesoangioblasts.

Second, Fig. 5a of Sampaolesi et al. ${ }^{1}$ indicates that muscle strength declined, rather than was maintained as they imply, in the treated legs of all dystrophic dogs in which it was assessed. At later time points, strength was always less than at 5 months. Between the ages of 5 and 9 months, the averaged decline in strength of the two older treated dogs was $\sim 40 \%$ ( $\sim 0.079$ to $\sim 0.048 \mathrm{~kg}^{-1}(\%)$ ), even more than the $\sim 25 \%$ decline in the control untreated dystrophic dog over the same period. Against this real $\sim 40 \%$ decline, purported improvements of $\sim 50 \%$ and $\sim 80 \%$ in treated leg-muscle strength relative to contralateral, untreated muscles of these two dogs (Fig. $5 \mathrm{~b}$ of Sampaolesi et al. ${ }^{1}$ ) are misleading and probably explained by simultaneous declines in contralateral muscle strength of $\sim 60 \%$ and $\sim 67 \%$, respectively.

Third, the dogs Valgus, Varus and Vaccin each received $5 \times 10^{7}$ mesoangioblasts per treatment, but these were infused into the aortic arch of Valgus and the left femoral artery of the others. At biopsy, $\sim 30-70 \%$ of fibres in sections of Valgus' left sartorius and gastrocnemius muscles appeared to be dystrophin-positive (Fig. 4a of Sampaolesi et al. ${ }^{1}$ ), whereas the equivalent dystrophin-positive proportion of Varus' left sartorius was $\sim 0-10 \%$, and that of Vaccin's left gastrocnemius was only $0-5 \%$. Numbers of mesoangioblasts reaching the lower left leg from upper aortic infusion, after major systemic blood diversions ${ }^{2}$, should have been $\sim 10$ times less than from direct femoral infusion. However, no assessed muscles of Valgus had fewer-and certainly not 10 times fewer-dystrophin-positive fibres than corresponding muscles of Varus or Vaccin. Moreover, some of the untreated muscles of Varus and Vaccin revealed up to $50 \%$ dystrophin-positive fibres (Fig. 4a of Sampaolesi et al. ${ }^{1}$ ).

Minimal mesoangioblast recirculation ${ }^{3}$ cannot easily explain these anomalies, whereas dystrophin-positive fibres in both treated and untreated muscles might represent false positives or revertants ${ }^{4}$, rather than evidence of mesoangioblast engraftment. Control biopsies from pre-treatment and untreated dystrophic dogs ${ }^{4}$ would have allowed these possibilities to be differentiated. Alternatively, if recirculation and engraftment is responsible for similar percentages of dystrophin-positive fibres in treated and 'untreated' tibialis cranialis muscles (Fig. 4a of Sampaolesi et al. ${ }^{1}$ ), then contrasting their strengths (Fig. 5b of Sampaolesi et al. ${ }^{1}$ ) is unfounded.

There is an indicator of benefit arising from this trial, although it is, perhaps, due to concurrent immunosuppression rather than to mesoangioblasts. Supplementary Fig. 7 of Sampaolesi et al. ${ }^{1}$ shows that levels of the muscle-breakdown marker serum creatine kinase decreased markedly soon after initiating immunosuppression (as previously seen in $m d x$ mice ${ }^{5}$ ) and before injection of heterologous mesoangioblasts, whereas in the days immediately after mesoangioblast injection, creatine kinase levels varied randomly, decreasing substantially $(>5,000 \mathrm{U})$ in three instances but increasing in five. This does not support the authors' hypothesis that creatine kinase reductions demonstrate mesoangioblast reconstitution of muscle fibres.

Control dystrophic dogs lived, on average, 129 days longer than six out of ten treated dogs (123 days longer than those treated with autologous mesoangioblasts). Three of the four remaining treated dogs, described as "well" 400 days post natal, "rapidly lost walking ability" when immunosuppression ceased (257 days post natal for two of these).

Altogether, the evidence presented by Sampaolesi et al. ${ }^{1}$ does not convince me that the dogs benefited from mesoangioblast treatment; a rigorous demonstration correlating muscle function, dystrophin expression and mesoangioblast infusion, with adequate controls, would have been helpful in this regard. It is therefore premature to consider a clinical trial in humans as a justifiable extension of this study.

\section{Allan H. Bretag ${ }^{1}$}

${ }^{1}$ Sansom Institute, School of Pharmacy and Medical Sciences, University of South Australia, Adelaide, South Australia 5000, Australia.

e-mail: a.bretag@unisa.edu.au

Received 3 January; accepted 17 October 2007.

1. Sampaolesi, M. et al. Mesoangioblast stem cells ameliorate muscle function in dystrophic dogs. Nature 444, 574-579 (2006).

2. Guyton, A. C. Textbook of Medical Physiology 7th edn, 230 (Saunders, Philadelphia, 1986).

3. Galvez, B. G. et al. Complete repair of dystrophic skeletal muscle by mesoangioblasts with enhanced migration ability. J. Cell Biol. 174, 231-243 (2006).

4. Dell'Agnola, C. et al. Hematopoietic stem cell transplantation does not restore dystrophin expression in Duchenne muscular dystrophy dogs. Blood 104, 4311-4318 (2004).

5. De Luca, A. et al. A multidisciplinary evaluation of the effectiveness of cyclosporine $A$ in dystrophic mdx mice. Am. J. Pathol. 166, 477-489 (2005).

doi:10.1038/nature06437

\section{Sampaolesi et al. reply}

Replying to: A. H. Bretag Nature 450, doi:10.1038/nature06437 (2007)

Bretag ${ }^{1}$ questions our finding ${ }^{2}$ that "dogs benefited from mesoangioblast treatment". We believe that this scepticism is not supported by careful examination of our data ${ }^{2}$.
Golden retriever dogs could not be matched for disease characteristics because they were chosen at 1 month of age, before the appearance of clinical symptoms. Indeed, the dog Vaccine, which did not 
show any clinical amelioration, had by chance the highest muscle contraction force at the beginning of treatment (Fig. 5a of ref. 2, green triangle). Dogs were encouraged in all cases: the videos of control dogs and of Vaccine lack audio but show the instructor encouraging the dogs with a caress, to which they react by swinging the tail and moving towards the instructor.

Our Fig. 5a (ref. 2) shows that, after a decrease between 5 and 9 months, contraction force increases again only in those dogs treated with donor cells; Fig. 5b of ref. 2, which compares treated and
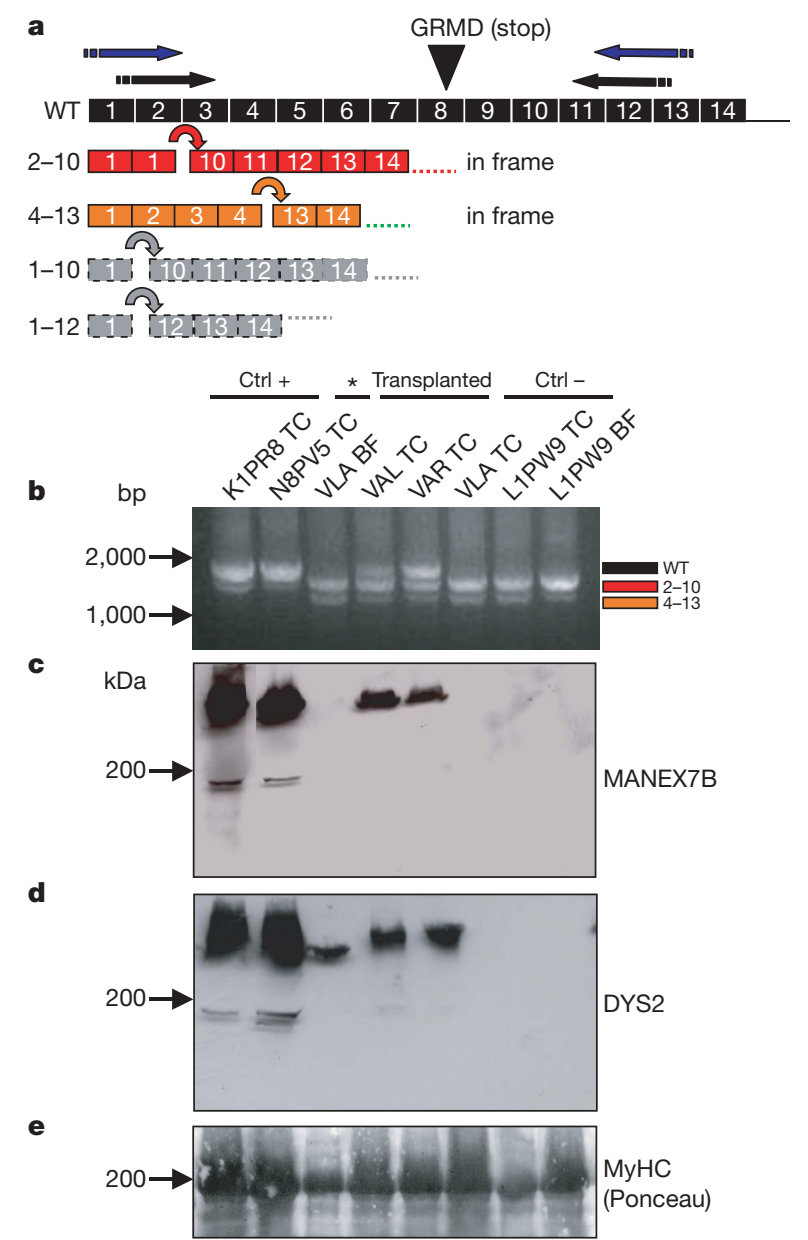

Figure 1 | Expression of wild-type dystrophin in dogs transplanted with wild-type mesoangioblasts. a, Four alternatively processed dystrophin gene transcripts can be amplified in golden retriever muscular dystrophy (GRMD) canine muscle tissue 9 . Only transcripts corresponding to exons 2-10 and 4-13 restore the correct reading frame. Wild type (WT, black) indicates the full-length dystrophin transcript; black and blue arrows indicate the position of oligonucleotides ${ }^{9}$ used for the nested polymerase chain reaction (PCR). b, Three transcripts are amplified by the nested RT-PCR analysis: the upper band corresponds to wild-type dystrophin (black), and the intermediate and lower bands correspond to the two inframe transcripts (red and orange, respectively). Note the presence of wildtype transcript in wild-type dogs (K1PR8 and N8PV5) and in dystrophic, transplanted dogs (VAL, Valgus; VAR, Varus), but not in untreated (Ctrl-: VLA and L1PW9) dystrophic dogs. BF, biceps femoralis; TC, tibialis cranialis. c, Western blot analysis using MANEX7B antibody (recognizing exons $7 / 8$, a gift from G. Morris) reveals wild-type dystrophin only in transplanted and wild-type dogs. d, Western blot analysis using DYS2 antibody (recognizing the carboxy terminus) reveals the wild-type dystrophin as above and a lower molecular mass protein in only one (asterisk; BF, biceps femoralis) muscle of Vlan (VLA), an untreated, dystrophic dog that shows a significant amount of revertant dystrophin without cell transplantation. e, Red Ponceau staining of myosin heavy chains of western blot shown in $\mathrm{d}$. untreated legs of the same animal, shows amelioration of contraction force in the three animals treated with donor cells and not in the one treated with autologous cells. In Duchenne muscular dystrophy, absence of dystrophin causes a reduced force of muscle contraction. We demonstrated a causal relation between dystrophin expression and force recovery, showing (Fig. 5c, d of ref. 2) that in the same muscle, dystrophin-positive fibres have normal force of contraction whereas dystrophin-negative fibres are as weak as untreated, dystrophic fibres ${ }^{2}$.

Contrary to Bretag's assertion ${ }^{1}$, Valgus received about $3 \times 10^{7}$ mesoangioblasts in the left femoralis artery and $2 \times 10^{7}$ in the left subclavian artery. Such a small difference $\left(3 \times 10^{7}\right.$ cells in the case of Valgus versus $5 \times 10^{7}$ cells in standard injections in the femoralis artery) cannot result in a marked difference in the number of dystrophin-positive fibres; the low numbers of dystrophin-positive fibres in Varus' sartorius may be due to more distal insertion of the catheter in the femoral artery, so that injected cells may not have reached the cranial part of the sartorius. Notably, we noticed and pointed out variable distribution of mesoangioblasts in the injected muscles. For this reason, we analysed more than 50 individual biopsies and found extensive, albeit variable, expression of dystrophin in treated dogs.

The presence of dystrophin in the contralateral muscles as well is not due to revertant fibres because an antibody directed against the mutated domain recognizes dystrophin in the muscles of transplanted dogs, confirming that wild-type dystrophin may only derive from wild-type donor cells. Previously unnoticed ${ }^{3}$, the biceps femoralis of one untreated dog shows a clear accumulation of revertant dystrophin, easily distinguishable from the wild-type protein (Fig. 1).

It is true that the first drop in serum creatine kinase for Valgus, Varus and Viko followed the first treatment with cyclosporine and is probably caused by the drug, but all the other declines occur after infusion of mesoangioblasts and in the continuous presence of the drug, so cannot be ascribed to it.

The cyclosporine effect is controversial, but Bretag ${ }^{1}$ quotes the only claim of benefit from cyclosporine $e^{4}$ and ignores others claiming deleterious $^{5-7}$ effects; moreover, the absence of any beneficial cyclosporine effect in bone-marrow-transplanted, dystrophic $\operatorname{dogs}^{8}$ is not mentioned.

Long-term survival was not a goal of this study, as immune suppression was suspended at the end of the experiment. Pathologists did not find any feature at autopsy that could have related to mesoangioblast accumulation.

Our results showed extensive dystrophin accumulation, clinical improvement and force preservation in the best available pre-clinical model of Duchenne muscular dystrophy. We are confident that our data are correct and form a suitable basis for future clinical experimentation.

Maurilio Sampaolesi ${ }^{1}$, Stephane Blot ${ }^{2}$, Roberto Bottinelli ${ }^{3} \&$ Giulio Cossu ${ }^{1}$ ${ }^{1}$ San Raffaele Scientific Institute, Stem Cell Research Institute, Via Olgettina 58, 20132 Milan, Italy.

e-mail: cossu.giulio@hsr.it

${ }^{2}$ Neurobiology Laboratory, Ecòle Veterinaire d'Alfort, 7 Avenue General de Gaulle, 94704 Maisons-Alfort, cedex, France.

${ }^{3}$ Department of Experimental Medicine University of Pavia, Via Forlanini 6-8, 27100 Pavia, Italy.

1. Bretag, A. H. Stem cell treatment of dystrophic dogs. Nature 450 , doi:10.1038/ nature06437 (2007).

2. Sampaolesi, M. et al. Mesoangioblast stem cells ameliorate muscle function in dystrophic dogs. Nature 444, 574-579 (2006).

3. Kornegay, J. et al. The cranial sartorius muscle undergoes true hypertrophy in dogs with golden retriever muscular dystrophy. Neuromuscul. Disord. 13, 493-500 (2003).

4. De Luca, A. et al. A multidisciplinary evaluation of the effectiveness of cyclosporine A in dystrophic mdx mice. Am. J. Pathol. 166, 477-489 (2005).

5. St-Pierre, S. J. G. et al. Glucocorticoid treatment alleviates dystrophic myofiber pathology by activation of the calcineurin/NF-AT pathway. FASEB J. 18, 1937-1939 (2004). 
6. Stupka, N. et al. The calcineurin signal transduction pathway is essential for successful muscle regeneration in mdx dystrophic mice. Acta Neuropathol. (Berl.) 107, 299-310 (2004).

7. Parsons, S. A. et al. Genetic disruption of calcineurin improves skeletal muscle pathology and cardiac disease in a mouse model of limb-girdle muscular dystrophy. J. Biol. Chem. 282, 10068-10078 (2007).
8. Dell'Agnola, C. et al. Hematopoietic stem cell transplantation does not restore dystrophin expression in Duchenne muscular dystrophy dogs. Blood 104, 4311-4318 (2004).

9. Schatzberg, S. J. et al. Alternative dystrophin gene transcripts in golden retriever muscular dystrophy. Muscle Nerve 21, 991-998 (1998).

doi: $10.1038 /$ nature 06438 